\title{
Drug eruptions
}

\author{
S.K. Goolamali \\ Central Middlesex Hospital, London and Northwick Park Hospital and Clinical Research Centre, Harrow, \\ Middlesex, UK.
}

\section{Introduction}

The last four decades have seen an enormous increase in the use of potent and toxic drugs as therapy. The considerable advances that these drugs have provided in the control and prevention of disease are matched by their potential to produce unwanted side effects many of which are reflected in the skin.

These side effects cannot in the main be predicted. Tests in animals may not reliably be extrapolated to the human and only occasionally can the occurrence of side effects be gauged from the structural formula of a new drug. With some fifty new drugs developed each year the practitioner is challenged simply to keep pace with their therapeutic indications. The results of one survey suggested that $30 \%$ of prescriptions from general practitioners was based on data supplied by drug companies. In 1985 it is also increasingly common to find that patients, when faced with a chronic disease have sought several medical opinions or have taken refuge in remedies which rely more on advertising than their therapeutic effectiveness. The available information suggests then that drug reactions are likely to be common.

In a recent study (Black \& Somers, 1984), drugrelated illness accounted for some $6 \%$ of hospital admissions in a year. It has been estimated that the average inpatient in a British hospital receives five drugs whilst one in an American hospital receives nine. Since a large majority of allergic reactions affect the skin the incidence of drug-induced cutaneous eruptions probably reflects the risk of allergic drug reactions in general. Reactions to drugs in over twenty thousand medical inpatients were studied in a large collaborative study (Arndt \& Jick, 1976). Skin reactions occurred in a little over $2 \%$. Despite the lack of data on non-hospitalized patients it is clear that even if only $2 \%$ of patients receiving drugs experience a ' $r a s h$ ' it is a number that we can all ill afford.

Correspondence: S.K. Goolamali, M.D., F.R.C.P., Northwick Park Hospital, Watford Road, Harrow, Middlesex HA1 3UJ, UK.

\section{Mechanisms of adverse drug reactions}

An adverse drug reaction is commonly defined as any response to a drug that is unintended and occurs at doses used in the prophylaxis or therapy of disease. Adverse reactions may occur as a result of inappropriate metabolism, idiosyncrasy or hypersensitivity to a drug. Impaired metabolism may result from altered oxidation or acetylation of a drug as may be seen in slow-acetylators in isoniazid-induced acne. An idiosyncratic reaction is an inbuilt abnormal response to a drug such as may occur when barbiturates or sulphonamides are given to a patient with porphyria. Hypersensitivity or allergy to a drug is mediated by an antigen-antibody reaction. Previous exposure and sensitization to the drug is a prerequisite though it is possible for a drug to induce antibody formation without clinical symptoms of hypersensitivity.

Hypersensitivity reactions are normally classified into four types (Gell \& Coombs, 1963) - Type 1 (immediate or anaphylactic), Type 2 (cytotoxic or autoallergic), Type 3 (immune complex disease) and Type 4 (cell-mediated or delayed). The vast majority of drug reactions are due to hypersensitivity and this review will be restricted to a brief discussion of these allergic responses and a description of some of the common skin reactions provoked by drugs.

\section{Type 1 reaction}

This is mediated by IgE or reagin. IgE antibodies are produced in response to a drug or drug-hapten complex and attach themselves preferentially to the mast cell. On exposure to antigen there is mediator release from the mast cell which then results in the well-described reactions of urticaria, angio-oedema, asthma and, in severe cases, anaphylaxis.

\section{Type 2 reaction}

This may present with thrombocytopenia, leucopenia or haemolytic anaemia. In this reaction the antigen (drug) combines with a platelet or red cell, for example, and stimulates the formation of antibodies to the drug-cell combination. Sedormid purpura, quinine 
purpura, many of the haemolytic anaemias, including that due to high dose penicillin therapy (Petz \& Fudenberg, 1966) and methyldopa, are examples of this type of reaction. Some 10 to $20 \%$ of patients receiving methyldopa develop a positive Coombs' test but only 0.5 to $1.0 \%$ develop a haemolytic anaemia. Approximately $70 \%$ of reported drug-induced immune haemolytic anaemias are a result of methyldopa. Leucopenia of immunological origin often with the presence of anti-leucocyte agglutinins is not infrequently a reaction of drugs.

\section{Type 3 reaction}

The initial exposure to the drug sensitizes and results in antibody, usually IgM or IgG, production. After an interval antigen-antibody complexes form and are deposited in the peripheral circulation. Complement is activated and causes accumulation of neutrophilic leucocytes which release lysosomal enzyme that destroys tissues. The commonly recognized systemic reaction in this category is the serum sickness syndrome. Fever, arthralgia and lymphadenopathy are typical features and classically arise from hypersensitivity to foreign serum. A similar clinical picture may result 1 to 3 weeks after treatment with penicillin, para-aminosalicylic acid or sulphonamides. Vasculitis, when a feature of the Type 3 reaction, is often attributed to sulphonamides but may also be caused by phenytoin, thiouracils, chlorpromazine, aspirin, gold and penicillin.

\section{Type 4 reaction}

This T-cell mediated or delayed hypersensitivity type of reaction is the basis of an allergic dermatitis often due to a topical medication. In a large European study (Bandmann et al., 1972), a third of the patients with an allergic contact dermatitis showed a hypersensitivity to a topical medicament. Sensitization is more likely to occur if the medication is applied to damaged skin. The importance of this 'contact' allergy is that it prohibits the systemic use of the same or related drug. If the offending drug is mistakenly taken it produces a widespread dermatitis. Amongst the contact sensitizers ethylenediamine hydrochloride used as a stabilizer in some creams is of particular importance. It cross-reacts with drugs used systemically such as aminophylline which consists of two-thirds theophylline and one-third ethylenediamine. Some antihistamines which are derived from ethylenediamine may also cause a generalized eruption if given to a sensitized patient (Fisher, 1976). Another recognized sensitizer is benzocaine found in anti-pruritic preparations, in local applications for the treatment of haemorrhoids and in some sunburn remedies. Benzocaine is a derivative of para-aminobenzoic acid
(PABA) and therefore may cross-react with othero PABA compounds such as procaine and dubucaine 3 Topical antihistamine, freely available as an over-the- $\frac{\mathbb{Q}}{2}$ counter preparation, is a commmon sensitizer as is $c$. neomycin especially when applied to chronic stasis dermatitis or otitis externa (Leyden \& Kligman, 1979)

\section{Types of cutaneous reactions}

Skin reactions to drugs are diverse. In a series of $4644^{\circ}$ cases during 1966-1970, Kuokkanen (1972) found $\vec{\circ}$ that exanthematous eruptions were the most commonand accounted for $46 \%$ of reactions. Urticariac occurred in $23 \%$ of cases, fixed drug eruption in $10 \%$;Oे erythema multiforme in some $5 \%$ and other reactions $5 \frac{O}{3}$ in less than $5 \%$ each. In another more recent study. (Kauppinen \& Stubb, 1984) exanthemas again formedthe largest group, $42 \%$ of 446 inpatients, fixed erup-N tions occurred in $21 \%$, urticaria-angioedema in $12.5 \%$. and erythema multiforme with Stevens-Johnson syndrome accounted for approximately $6 \%$. The remain-o der comprised eczema, toxic epidermal necrolysis,, photosensitivity reactions, purpura and the systemic $O$ lupus erythematosus (SLE) syndrome.

\section{Exanthematous eruption}

This type of reaction can be caused by almost a drug. Itch may or may not be associated and differentiation from a viral exanthem may at times be difficuft. The greatest incidence occurs with the penicillins and the eruption commonly consists of maculo-papularo erythematous lesions. The risk of developing penicillin $\bar{\varnothing}$ hypersensitivity is increased in patients with a previous history of other drug allergy and in those of whom the drug is given parenterally. In one study (Shapiro et al., $\stackrel{\Im}{\supset}$ 1969) a rash was reported in $4.5 \%$ of 622 patients treated with penicillins other than ampicillin, in $9.5 \%$ of 422 patients treated with ampicillin and in $1.8 \%$ of 3 2941 patients not receiving either of these drugs.Although ampicillin may provoke urticaria, it com- 3 . monly causes a morbilliform eruption (Figure 1) which lasts a few days. An increased incidence of 3 ampicillin rash occurs in infectious mononucleosis, $O$ cytomegalovirus mononucleosis, lymphatic leukaemia and in viral respiratory infections (Almeyda \& Levantine, 1972). In these conditions the rash may also be somewhat prolonged.

Other drugs commonly associated with an exanth- $N$ ematous eruption include barbiturates, sulphon- $N$ amides and allied anti-diabetic and diuretic drugs, $\omega$ phenytoin, erythromycin, allopurinol and gold salts. Gold salts were first used in France in the 1920s and $\stackrel{\circ}{\circ}$ 1930s though their efficacy as an anti-rheumatic drug $\stackrel{\Phi}{\oplus}$ was confirmed very much later (Fraser, 1945). Two forms of these drugs are in common use, the aqueous 


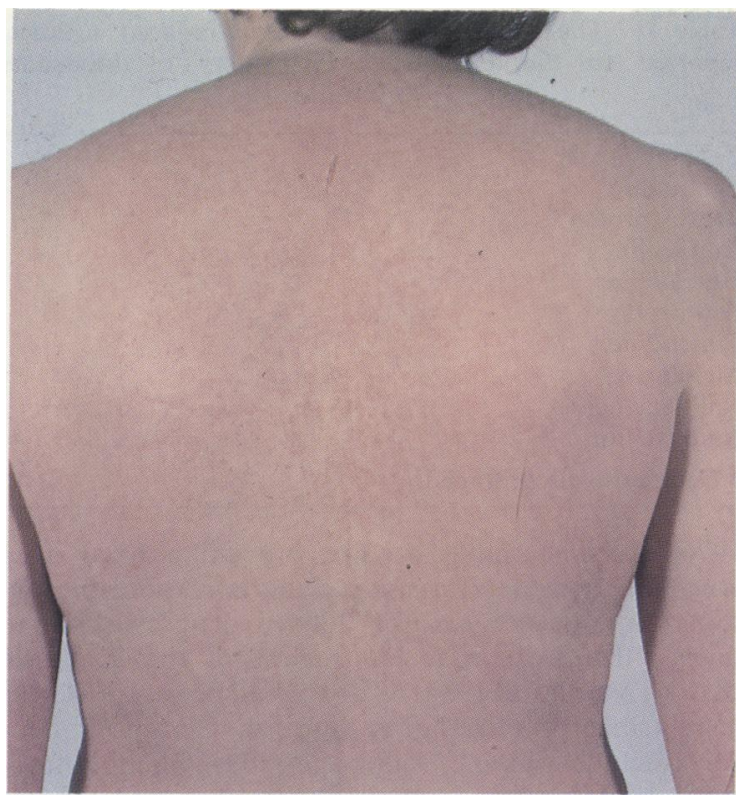

Figure 1 Ampicillin reaction.

based sodium aurothiomalate and the oil based aurothioglucose. Both drugs are administered intramuscularly. Overall, approximately $30 \%$ of patients started on chrysotherapy may, at least temporarily, have to discontinue the drug because of side effects, the most common being a rash or mucous membrane lesions. The most common eruption is erythematous, maculo-papular and pruritic, but a lichen-planus like eruption or a pityriasis rosea-like rash also occurs (Penneys et al., 1974). After the reaction has settled gold therapy can generally be

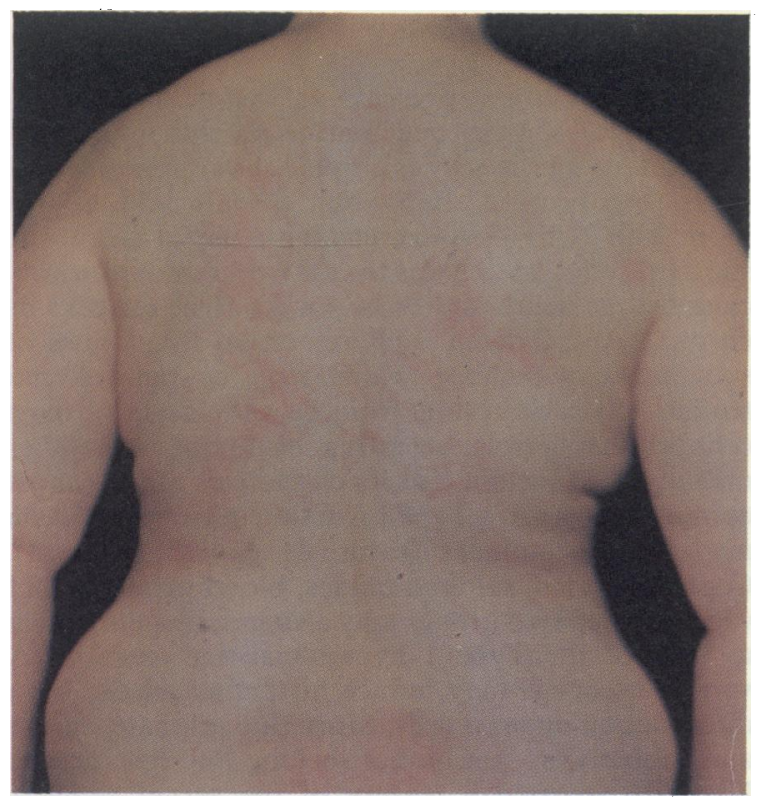

Figure 2 Penicillin induced urticaria.

restarted without incident (Klinefelter, 1975).

Allopurinol, an analogue of hypoxanthine, is an inhibitor of xanthine oxidase and is used widely in the prophylaxis of gout. Its major side effect is an erythematous maculopapular eruption though occasionally urticaria and toxic epidermal necrolysis have been seen. A drug rash has been noted in some $2 \%$ of patients taking allopurinal alone (Boston Collaborative Drug Surveillance Program, 1972) but the incidence rose to $22.4 \%$ in patients treated simultaneously with both allopurinol and ampicillin.

Table I Hypersensitivity drug reactions

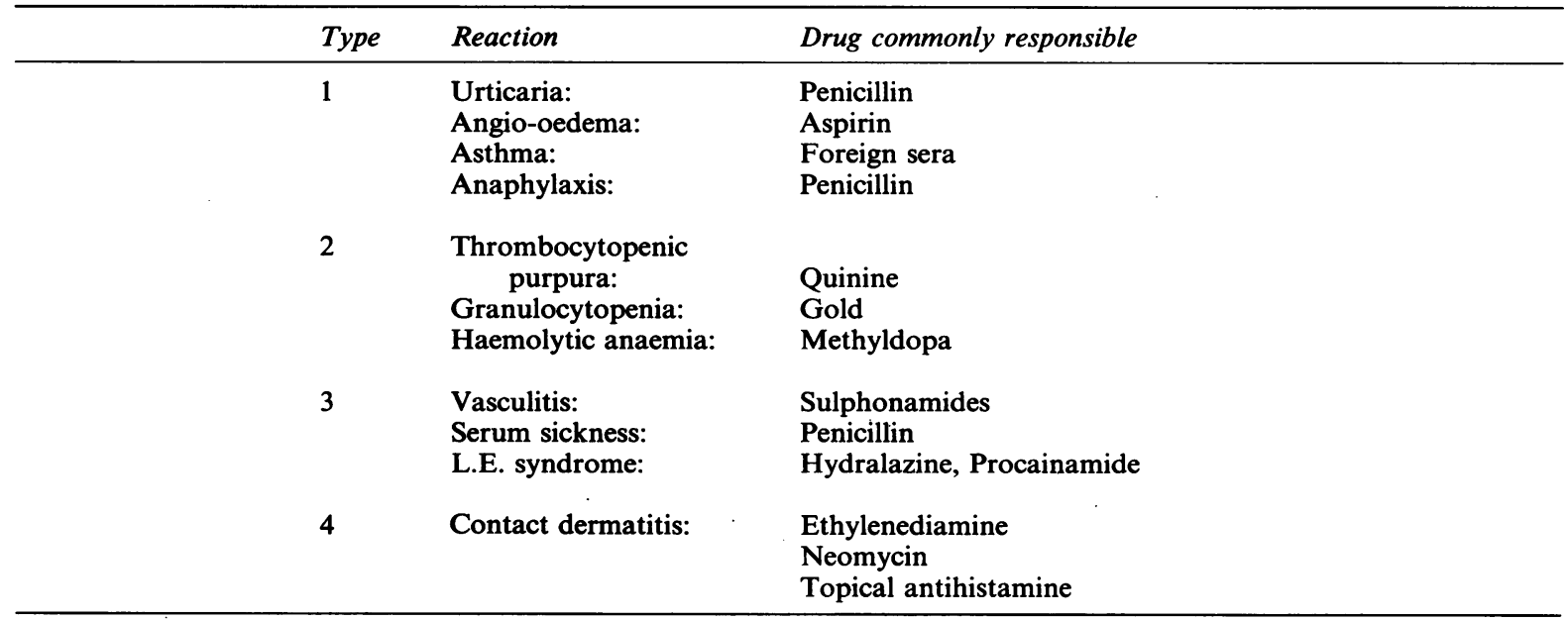




\section{Urticaria and angioedema}

Urticaria or 'hives' presents as well-defined, erythematous, raised often oedematous patches in the skin. The lesions are usually multiple, of variable size and invariably pruritic. Individual wheals seldom last more than 24 to 48 hours which is a useful diagnostic sign. Episodes lasting less than 6 weeks are arbitrarily classified as acute and those longer than 6 weeks as chronic. Angioedema is characterized by plaques of subcutaneous oedema which may be painful and tender. Urticaria results from degranulation of mast cells in the dermis, releasing histamine and other mediators. Penicillin and its derivatives are the most common causes of a Type 1 reaction in which urticaria may be a prominent feature (Figure 2, Table I). Cephalosporins, sulphonamides, blood products and vaccines prepared in eggs may also induce an urticaria as part of the Type 1 hypersensitivity reaction. A variety of drugs may induce histamine release by a direct action on mast cells. Morphine sulphate, curare and polymyxin antibiotics belong to this group. Urticaria is often a feature of the serum sickness syndrome, and, finally, aspirin and non-steroidal anti-inflammatory agents may induce urticaria (Ros $e t$ al., 1976). It is postulated that these drugs inhibit prostaglandin production and the lack of the inhibitory action of prostaglandins allows the development of urticaria.

In a 17 year prospective study of the clinical characteristics of patients with a history of allergy to aspirin (Speer et al., 1981), the most common manifestation was urticaria-angioedema. In these patients $90 \%$ were also sensitive to inhalants $(76 \%)$, foods . $(74 \%)$ and other drugs $(43 \%)$. The ten most common drugs which may induce urticaria as reported to the Committee on Safety of Medicines in the United Kingdom between the years 1964 and 1983 (Griffin, 1983) are shown in Table II.

\section{Fixed drug eruption}

The term fixed drug eruption (l'eruption fixe) was introduced by Brocq (1894) when he described three patients in whom an erythematous pigmented eruption developed after ingesting antipyrine. It usually presents as a solitary pruritic erythematous macule which forms into an oedematous plaque. Vesicles and bullae may develop later. When the acute phase subsides there is residual pigmentation which becomes more prominent with each exposure to the causative drug. With repeated attacks existing lesions increase in size and new lesions appear. The lesions may appear anywhere on the body though the lips, hands and glans penis (Figure 3 ) are commonly affected. The length of time from re-exposure to a drug and onset of symptoms varies between half an hour to 8 hours (Stubb,
Table II The top ten causes of drug-induced urtica年a reported to the Committee on Safety of Medicin 1964-1983 (Griffin, 1983)

Cotrimoxazole

Fenbufen

Benoxaprofen*

Ampicillin

Fenclofenac

Feprazone

Cimetidine

Alclofenac*

Nalidixic acid

Amoxycillin

* Drug now discontinued.

1976) Antihistamines do not prevent a fixed drîg eruption. In general a single drug is responsible bुㅐ some patients may exhibit a fixed drug eruption to several drugs. In a recent review of the literatume (Korkij \& Soltani, 1984) 68 drugs had been reported responsible for a fixed drug eruption. Common offetsders include quinine, sulphonamides, phenolphthalein, barbiturates, oxyphenbutazone and chlordiazepoxide. Phenolphthalein is considered a safe and reliable drug and hence found in proprietary pof gatives, and because it is tasteless it is included in thoge sold in the form of chewing gum or chocolate.

The mechanism which determines the localizक्षिi $\overrightarrow{\mid r}$

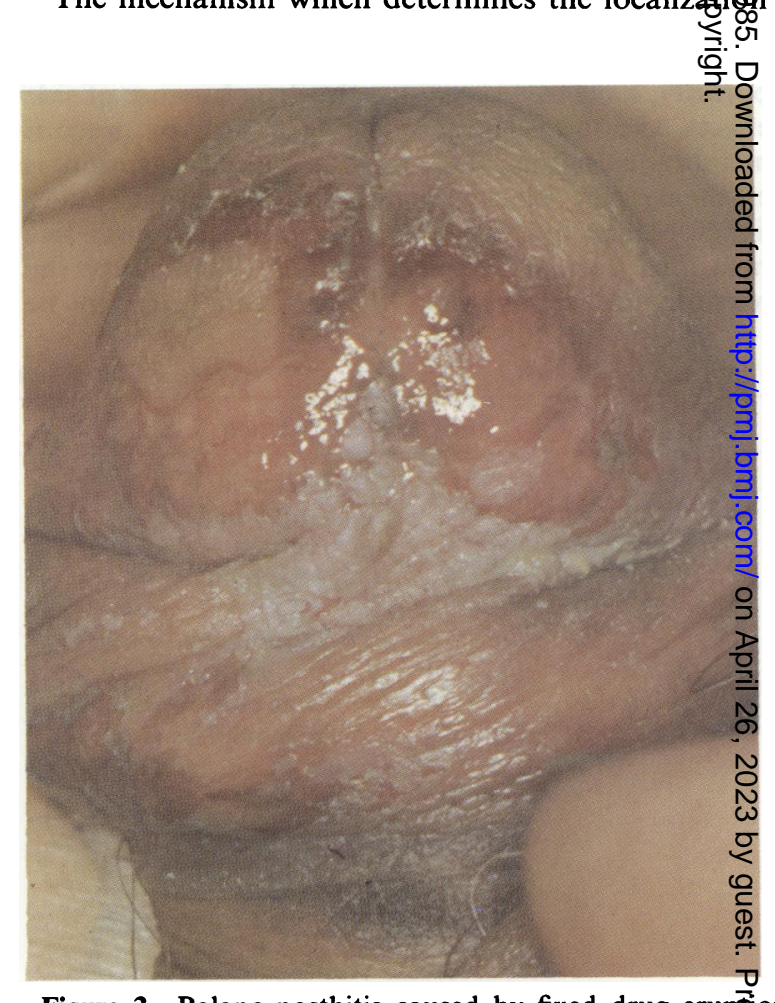

Figure 3 Balano-posthitis caused by fixed drug eruppion showing lesions. 
of the skin lesions remains unexplained. Patch testing on unaffected skin with the drug responsible for the fixed eruption has invariably produced negative results whilst occasionally a positive response has been obtained when the patch test has been applied to previously affected but 'healed' skin (Welsh, 1961). A blood-borne mediator has been identified as a possible cause in a phenolphthalein-induced fixed eruption (Wyatt et al., 1972) but this fails to explain why the lesions are so localized.

\section{Erythema multiforme}

The diagnostic lesion is the iris or target lesion (Figure 4). As the name multiforme implies it may present with several different types of lesions. These may consist of urticated papules or, as in the severe form of the disease, bullae, which become widespread and are associated with ulceration of the oral and genital mucosa-the Stevens-Johnson syndrome.

Erythema multiforme is considered to be a hypersensitivity reaction commonly to a drug or to an underlying virus or bacterial infection. The commonest associations are with preceding herpes simplex or mycoplasma infection. Streptococcal infection, infectious mononucleosis, tuberculosis, sarcoidosis, X-ray therapy of malignant disease and sometimes malignancy itself are other recognized causes. The most common drug causes of erythema multiforme are sulphonamides, phenytoin, barbiturates, phenylbutazone, sulphonylureas, penicillin and salicylates.

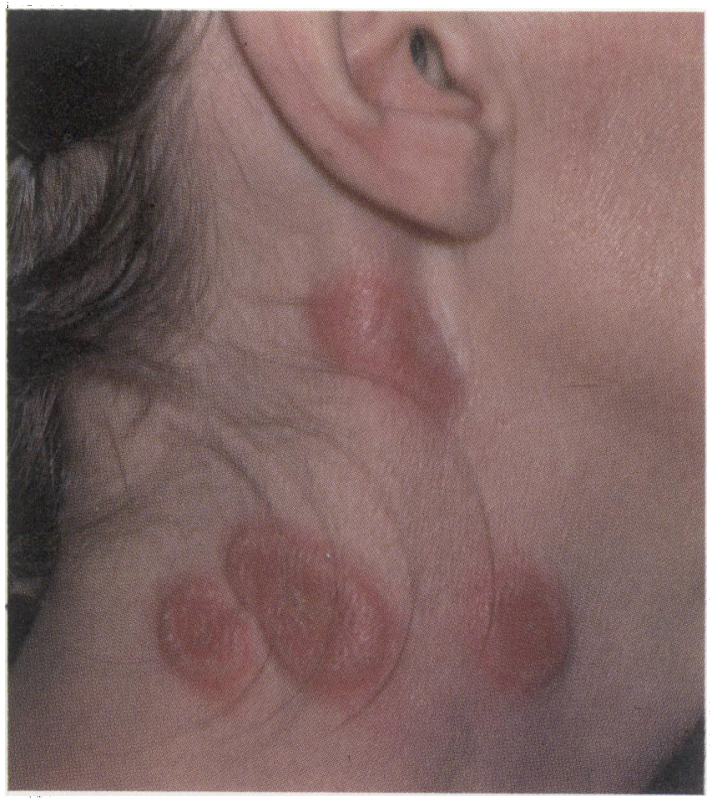

Figure 4 Erythema multiforme.
In a recent review of the literature 40 drugs had been implicated as the cause of erythema multiforme (Huff et al., 1983). Sulphonamides were the most frequent offenders. Sulphonamide-associated erythema multiforme usually appears a week or two after therapy but may occur within hours in patients who have previously been sensitized to the drug. Stevens-Johnson syndrome may also be caused by the long acting sulphonamides (Carroll et al., 1966), penicillins, diphenylhydantoin and chlorpropamide (Bianchine et al., 1968).

\section{Photosensitivity}

Photosensitivity is an all-encompassing term to describe untoward reactions to non-ionizing radiation. There are two main types - phototoxicity and photoallergy. A phototoxic reaction relies on a high concentration of a photosensitizing agent in the skin and occurs when a sufficient quantity of light of appropriate wavelength reaches the skin. Everyone is capable of exhibiting a phototoxic reaction which presents as erythema, urticaria and oedema within 24 hours of light exposure. The eruption is confined to light exposed areas commonly the face, $\mathrm{V}$ area of neck, dorsum of the hands and the feet. Acute erythema is usually followed by hyperpigmentation. A phototoxic reaction may occur on first administration and subsides quickly on withdrawal of the drug. Drugs recognized as causes include the tetracycline group especially demethylchlortetracycline, nalidixic acid, chlorothiazide, sulphonamides and griseofulvin.

A photoallergic reaction is less common and may occur with small quantities of a drug. Photoallergy may be of the 'immediate' type and then commonly presents as solar urticaria or produces an eczema as a Type 4 reaction (Horio, 1984). The latter variety is the more frequently encountered. The eruption, unlike the phototoxic reaction, may spread to areas which have not been light exposed. Normally there is a delay of 48 hours before the onset of the reaction. Although drugs administered systemically will induce photoallergy it is important to recognize that a photoallergic dermatitis may be caused by a topically applied photosensitizer. In practice it may be difficult to differentiate between a phototoxic and a photoallergic reaction in an individual patient especially as similar drugs produce both types of reaction. Those drugs which may cause photoallergy include chlorpromazine, chlorothiazide, sulphonamides and griseofulvin.

\section{LE syndrome}

A syndrome clinically indistinguishable from systemic lupus erythematosus may be provoked by a variety of drugs. Those commonly identified include hydralazine, procainamide, isoniazid and phenytoin. 
The development of SLE with these drugs appears to be dose related and with hydralazine a dose greater than $200 \mathrm{mg} / \mathrm{d}$ ( $300 \mathrm{mg}$ in rapid acetylators) is more likely to induce the syndrome.

The syndrome may present with a variety of skin signs which include erythema with the familiar 'butterfly' rash, urticaria, purpura, photodermatitis, alopecia, livedo reticularis, cutaneous vasculitis, hyperpigmentation and nail fold telangiectasia. Arthralgia and pleurisy also occur but renal and central nervous system involvement is uncommon. Tests for antinuclear factor are invariably positive and LE cells are commonly found. Antibodies to single strandedDNA may be found but those against double stranded DNA are rare. A genetic predisposition to the syndrome has been claimed because the HLA antigen DRW-4 is commonly identified in patients. Indeed the combination of HLA-DRW-4, female sex, slow acetylator status and a minimum dose of $200 \mathrm{mg} / \mathrm{d}$ of hydralazine almost inevitably led to the syndrome in one study (Batchelor et al., 1980). Other drugs known to provoke the syndrome are griseofulvin, methyldopa, chlorpromazine, para-aminosalicylic acid and thiouracil.

\section{Lichen planus-like drug eruption}

Several drugs produce an eruption identical with or virtually indistinguishable from lichen planus (Figure 5). Lesions in the mouth are more frequent in 'true' lichen planus but the distribution of the eruption over the body is similar in the two varieties. In addition the lesions in both types heal with pigmentation, and histology, in most cases, cannot separate a druginduced lichen planus from that which arises de novo. Gold and organic arsenicals were amongst the first to be recognized as drugs which produced a lichenoid rash. Arsenic is now considered outdated as a therapeutic measure though gold has gained in popularity in recent years for the treatment of rheumatoid arthritis. Mepacrine was found to induce a lichenoid eruption as long ago as the Second World War when troops who took the drug as an antimalarial agent developed the typical rash. Chloroquine will also cause a lichenoid eruption and thiazide diuretics, chlorothiazide and hydrochlorothiazide (Harber et al., 1959) are known to cause a photosensitive lichenoid eruption. Amiphenazole, a respiratory stimulant, may cause bone marrow depression with long term use but lichenoid eruptions may also be induced. Methyldopa includes in its potential side effects a lichenoid rash.

\section{Drug-induced alopecia}

Hair growth is normally divided into three stages: telogen when the hair is resting, anagen when it is

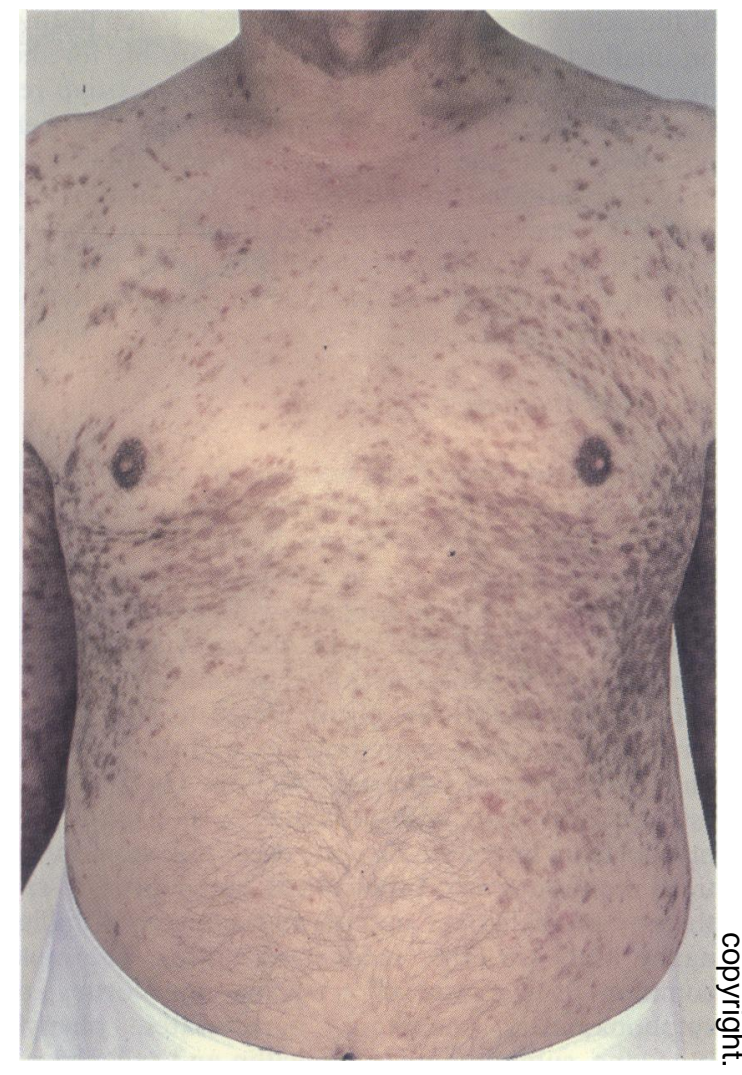

Figure 5 Drug-induced lichen planus.

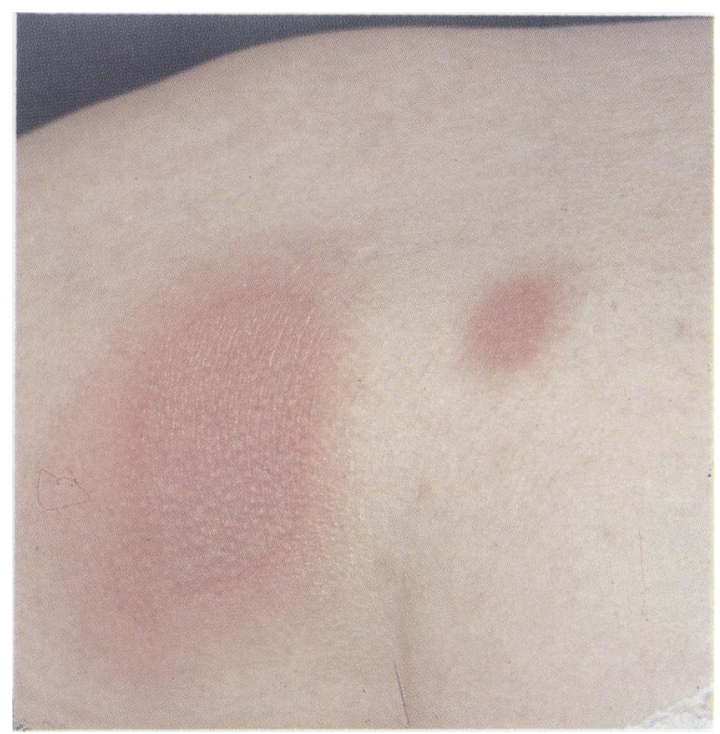

Figure 6 Fixed drug eruption. 
actively growing and catagen when it is involuting. In the adult, approximately $85 \%$ of the scalp hair is in anagen, $14 \%$ in telogen and $1 \%$ in catagen. With hair loss associated with the contraceptive pill, the telogen hairs are shed - a phenomenon known as telogen effluvium. Acute disease or emotional stress may provoke a similar hair loss. With cyclophosphamide therapy some anagen follicles enter catagen prematurely and inhibition of mitosis results in a constriction of the hair shaft. These hairs may be shed 4-6 days after the first effective dose. Anti-cancer drugs commonly cause alopecia of the scalp though with long term use of chemotherapeutic agents there may also be loss of axillary and pubic hair. Severe loss is common with cyclophosphamide, the nitrosoureas (e.g. lomustine) and doxorubicin. The hair returns on stopping the drug though occasionally it is of a different colour and texture than previously (Falkson \& Schulz, 1981). Heparin, the coumarins and indandiones all cause a telogen effluvium 2 to 3 months after starting anticoagulant therapy. Antithyroid drugs, thiouraeils and carbimazole, may also induce alopecia. Loss of scalp hair occurred in five women who were given carbimazole in doses varying between 15 and $60 \mathrm{mg} / \mathrm{d}$ for 4 to 40 weeks. All improvéd when carbimazole was discontinued or the dose reduced (Papadopoulos \& Harden, 1966).

\section{Drug-induced hyperpigmentation}

The antimalarials cause pigmentary changes in some $25 \%$ of patients receiving these drugs for more than 3 to 4 months (Levantine \& Almeyda 1973). Mepacrine produces a diffuse yellowish discolouration of the skin. It occurs in most patients who receive the drug and fades a few weeks or several months after therapy is discontinued. Chloroquine may whiten hair and at the same time stain the skin a bluish-grey colour. Prolonged high doses of chlorpromazine and related phenothiazines produce hyperpigmentation, particularly in sun-exposed areas of skin (Satanove, 1965). The pigmentation is cumulative and fades only a little in winter. The skin colour changes range from $\tan$ in the early stages to slate-grey later and finally to purple. Minocycline, a tetracycline derivative, used in the treatment of acne vulgaris, may cause patchy or diffuse pigmentation normally described as blue-black in colour (McGrae \& Zelickson, 1980; Simons \& Morales, 1980).

Chloasma is a well recognized side effect of oral contraceptives. In one study some 3 of 10 patients receiving the drug developed the pigmentation (Resnik, 1967). Discontinuation of the oral contraceptive may cause partial remission in pigment though in many the chloasma remains unchanged.

Hyperpigmentation is also a well recognized com- plication of several anti-cancer drugs. Those commonly reported include bleomycin, busulphan, cyclophosphamide, systemic fluorouracil, hydroxyurea and mithramycin. The hyperpigmentation associated with these drugs appears to be unrelated to increased ACTH or melanocyte stimulating hormone activity. Bleomycin induced pigmentation occurs in approximately $30 \%$ of patients receiving the drug and may be diffuse, patchy or linear. The patchy pigmentation is often prominent on pressure areas - the elbows, knees and buttocks (Blum et al., 1973). Long term therapy with busulphan can produce a diffuse pigmentation akin to Addison's disease (Harrold, 1966). Cyclophosphamide hyperpigmentation may be widespread (Harrison \& Wood, 197.2) or localized to the palms soles or nails (Shah et al., 1978). Mithramycin produces hyperpigmentation in $35 \%$ of patients on the drug whilst long term treatment with hydroxyurea induces both alopecia and pigmentation (Kennedy et al., 1975).

\section{Where are we after 60 years?}

Some sixty years ago Henry Semon in his article on Drug Eruptions in this Journal (Semon, 1926) described the problem of halogen acne, the lesions which arsenic may induce, the use of mercury ointment as treatment of pediculosis pubis and the development of idiosyncrasy to aspirin and quinine. In 1985 only the latter two drugs survive as therapeutic agents. The diagnosis of a drug eruption is no easier today than it was six decades ago. In many ways it is more difficult with the profusion of drugs and plethora of recognized side effects. Penicillin allergy can now be reliably confirmed by 'scratch' and 'prick' skin tests and intradermal techniques using benzylpenicilloyl polylysine conjugate and a mixture of minor determinants of penicillin (Chandra et al., 1980). Patch testing is valuable in the diagnosis of a contact dermatitis but can give misleading results when used to diagnose reactions not of the cell mediated-delayed hypersensitivity type. Laboratory tests otherwise have not provided a fail-safe method of isolating a single drug from many as the cause of a specific drug reaction.

In the main the best the modern clinician can do is to suspect a drug and reproduce the rash by reintroducing the drug, the so called provocation test. In practice this is not always possible nor desirable. The physician is often forced to react to the eruption as an amateur sleuth who has been invited to decide the odds on a particular suspect drug as the cause of the cutaneous offence. Clearly there is urgent need for research into the vexed problem of drug eruptions which occasionally cause death, undoubtedly increase the length of inpatient stay for a considerable number of patients and are a source of physical and psychological discomfort for many. 
References

ALMEYDA, J. \& LEVANTINE, A. (1972). Drug reactions XIX. Adverse cutaneous reactions to the penicillins-ampicillin rashes. British Journal of Dermatology, 87, 293.

ARNDT, K.A. \& JICK, H. (1976). Rates of cutaneous reactions to drugs. A report from the Boston Collaborative Drug Surveillance Program. Journal of the American Medical Association, 235, 913.

BANDMANN, H.J., CALNAN, C.J., CRONIN, E., FREGERT, S., HJORTH, N., MAGNUSSON, B., MAIBACH, H., MALTEN, K., MENEGHINI, C.L., PIRILA, V. \& WILKINSON, D.S. (1972). Dermatitis from applied medicaments. Archives of Dermatology, 106, 335.

BATCHELOR, J.R., WELSH, K.I., MANSILLA TINOCO, R., DOLLERY, C.T., HUGHES, G.R., BERNSTEIN, R., RYAN, P., NAISH, P.F., ABER, G.M., BING, R.F. \& RUSSELL, G.I. (1980). Hydralazine-induced systemic lupus erythematosus: influence of HLA-DB and sex on susceptibility. Lancet, i, 1107.

BIANCHINE, J.R., MACARAEG, P.V.J., LASAGNA, L., AZARNOFF, D.L., BRUNK, F., HVIDBERG, E.F. \& OWEN, J.A. (1968). Drugs as etiologic factors in the Stevens-Johnson syndrome. American Journal of Medicine, 44, 390.

BLACK, A.J. \& SOMERS, K. (1984). Drug related illness resulting in hospital admission. Journal of the Royal College of Physicians of London, 18, 40.

BLUM, R.H., CARTER, S.K. \& AGRE, K. (1973). A clinical review of bleomycin- a new antineoplastic agent. Cancer, 31, 903.

BOSTON COLLABORATIVE DRUG SURVEILLANCE PROGRAM. (1972). Excess of ampicillin rashes associated with allopurinol or hyperuricaemia. New England Journal of Medicine, 286, 505.

BROCQ, L. (1894). Eruption érythemato-pigmentée fixe due à l'antipyrine. Annales de Dermatologie et de Syphiligraphie (Paris) 5, (Series 3), 308.

CARROLL, O.M., BRYAN, P.A. \& ROBINSON, R.J. (1966). Stevens-Johnson syndrome associated with long-acting sulphonamides. Journal of the American Medical Association, 195, 691.

CHANDRA, R.K., JOGLEKAR, S.A \& TOMAS, E. (1980). Pencillin allergy:antipenicillin IgE antibodies and immediate hypersensitivity skin reactions employing major and minor determinants of penicillin. Archives of Disease in Childhood, 55, 857.

FALKSON, G. \& SCHULZ, E.J. (1981). Changes in hair pigmentation associated with cancer chemotherapy. Cancer Treatment Reports, 65, 529.

FISHER, A.A. (1976). Allergic dermatitis medicamentosa. The systemic contact-type variety. Cutis, 18, 637.

FRASER, T.N. (1945). Gold treatment in rheumatoid arthritis. Annals of the Rheumatic Diseases, 4, 71.

GELL, P.G.H. \& COOMBS, R.R.A. (1963). In Clinical Aspects of Immunology, 1st Edition. Blackwell Scientific Publications: Oxford.

GRIFFIN, J.P. (1983). Drug induced allergic and hypersensitivity reactions. Practitioner, 227, 1283.

HARBER, L.C., LASHINSKY, A.M. \& BAER, R.L. (1959). Skin manifestations of photosensitivity due to chlorothiazide and hydrochlorothiazide Journal of Investigative Dermatology, 33, 83 .

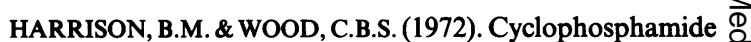
and pigmentation. British Medical Journal, 2, 352.

HARROLD, B.P. (1966). Syndrome resembling Addison's disease following prolonged treatment with busulfan $\stackrel{0}{\rightarrow}$ therapy, British Medical Journal, 1, 463.

HORIO, T. (1984). Photoallergic reaction: classification and $\frac{\bar{\sigma}}{\mathrm{O}}$ pathogenesis. International Journal of Dermatology, 23, $\overline{\bar{\alpha}}$ 376.

HUFF, J.C., WESTON, W.L. \& TONNESEN, M.G. (1983). Erythema multiforme: a critical review of characteristics, diagnostic criteria and causes. Journal of the American $\overrightarrow{0}$ Academy of Dermatology, 8, 763.

KAUPPINEN, K. \& STUBB, S. (1984). Drug eruptions: $\vec{\omega}$ causative agents and clinical types. Acta Dermatologica Venereologica (Stockholm), 64, 320.

KENNEDY, B.J., SMITH, L.R. \& GOLTZ, R.W. (1975). Skin 3 changes secondary to hydroxyurea therapy. Archives of $\sigma$ ) Dermatology, 111, 183.

KLINEFELTER, H.F. (1975). Reinstitution of gold therapy in N rheumatoid arthritis after mucocutaneous reactions. Journal of Rheumatology, 2, 21.

KORKIJ, W. \& SOLTANI, K. (1984). Fixed drug eruption: a brief review. Archives of Dermatology, 120, 520.

KUOKKANEN, L. (1972). Drug eruptions: A series of $464-$ cases in the Department of Dermatology, University of $\bigcirc$ Turku, Finland, during 1966-1970. Acta Allergologica, $27,407$.

LEVANTINE, A. \& ALMEYDA, J. (1973). Drug induced changes in pigmentation. British Journal of Dermatolog 89, 105.

LEYDEN, J.J. \& KLIGMAN, A.M. (1979). Contact dermati霝 to neomycin sulphate. Journal of the American Medicăt Association, 242, 1276.

MCGRAE, J.D. \& ZELICKSON, A.S. (1980). Skin pigmentation $\bar{\partial}$ secondary to minocycline therapy, Archives of Der- 응 matology, 116, 1262.

PAPADOUPOULOS, S. \& HARDEN, McG. R. (1966). Hair loss $\overrightarrow{\vec{O}}$ in patients treated with carbimazole. British Medical 윽 Journal, 2, 1502.

PENNEYS, N.S., ACKERMAN, A.B. \& GOTTLIEB, N.L. (1974). Gold dermatitis. A clinical and histopathological study. Archives of Dermatology, 109, 372.

PETZ, L.D. \& FUDENBERG, G.H.H. (1966). Coombs positive hemolytic anaemia caused by penicillin administration. New England Journal of Medicine, 274, 171.

RESNIK, S. (1967). Melasma induced by oral contraceptive $\delta$ drugs Journal of the American Medical Association, 199, $३$ 601.

ROS, A.M., JUHLIN, L. \& MICHAELSSON, G. (1976). A follow-up study of patients with recurrent urticaria and hypersensitivity to aspirin, benzoates and azo dyes. British Journal of Dermatology, 95, 19.

SATANOVE, A. (1965). Pigmentation due to phenothiazines in high and prolonged dosage. Journal of the American Medical Association, 191, 263.

SEMON, H.C. (1926). Drug eruptions. Postgraduate Medical Journal, 1, 144.

SHAH, P.C., RAO, K.R.P. \& PATEL, A.R. (1978). Cyclophosphamide induced nail pigmentation. British Journal of Dermatology, 98, 675 . 
SHAPIRO, S., SISKIND, D.V., SLONE, D., LEWIS, G.P. \& JICK, H. (1969). Drug rash with ampicillin and other penicillins. Lancet, ii, 969.

SIMONS, J.C. \& MORALES, A. (1980). Minocycline and generalized cutaneous pigmentation. Journal of the American Academy of Dermatology, 3, 244.

SPEER, F., DENISON, T.R. \& BAPTIST, J.E. (1981). Aspirin allergy. Annals of Allergy, 46, 123.

STUBB, S. (1976). Blood leucocytes with special reference to basophils and eosinophils during provocation tests in fixed eruption and drug exanthema. Acta Dermatologica Venereologica, 56, 1.

WELSH, A.L. (1961). The fixed eruption. Charles Thomas: Springfield, Illinois.

WYATT, E., GREAVES, M.W. \& SONDERGAARD, J. (1972). Fixed drug eruption (phenolphthalein): evidence for a blood borne mediator. Archives of Dermatology, 8, 763. 